\title{
Aplicando o SPIDe à Engenharia de Requisitos de um Ambiente Virtual de Aprendizagem: resultados preliminares
}

\author{
Fiama S. Silva ${ }^{1}$, Jean C. S. Rosa ${ }^{2}$, Gilton J. F. Silva ${ }^{1}$, Ecivaldo Matos ${ }^{2}$ \\ ${ }^{1}$ Departamento de Computação - Universidade Federal de Sergipe (UFS) \\ Av. Marechal Rondon, s/n - São Cristóvão - SE - Brasil \\ ${ }^{2}$ Departamento de Ciência da Computação - Universidade Federal da Bahia (UFBA) \\ Av. Adhemar de Barros, s/n - Salvador - BA - Brasil \\ fssantos@dcomp.ufs.br, jean.rosa@ufba.br, gilton@dcomp.ufs.br, \\ ecivaldo@ufba.br
}

Resumo. Entender os desejos e necessidade dos (potenciais) usuários são desafios para a Engenharia de Requisitos e para o Design de Interação. Existem métodos, técnicas, modelos, ferramentas para auxiliar designers de interação a identificar e especificar esses desejos e necessidades, dentre eles o SPIDe (Semio-Participatory Interaction Design). O SPIDe é um framework para o design de interação baseado na perspectiva do Design para/por Todos. No SPIDe, os usuários assumem o papel de codesigners de interação. Este artigo apresenta resultados preliminares sobre a aplicação do SPIDe integrado a Engenharia de Requisitos de um Ambiente Virtual de Aprendizagem.

Abstract. Understanding users' desires and needs are challenges to Requirements Engineering and Interaction Design. There are methods, techniques, models, tools to help interaction designers identify and specify those desires and needs, among them the Semio-Participatory Interaction Design (SPIDe) framework. SPIDe is a framework for interaction design based on Design for/by All. In SPIDe, users assume the role of interaction codesigners. This paper presents preliminary results on the application of SPIDe integrated to Requirements Engineering of a Virtual Learning Environment.

\section{Introdução}

O software educacional é um artefato computacional que deve atuar como mediador em atividades de formação e desenvolvimento de pessoas em áreas distintas do conhecimento (Gomes e Wanderley, 2003). A utilização desses artefatos computacionais tem favorecido a construção do conhecimento. Todavia, é necessário atentar-se para a sua qualidade, isto para que os seus benefícios sejam adequados (Junior e Paola, 2014).

A qualidade de softwares educacionais está relacionada com a capacidade em que ele pode atender os desejos e necessidades dos seus usuários com relação ao ensino e a aprendizagem, sendo os usuários mais característicos desse tipo de softwares os (as) professores(as) e estudantes (Gomes e Padovani, 2005). Kujala et al. (2001) abordam 
que além dos desejos e necessidade, o contexto de uso também deve ser considerado, de modo que a análise dessas características deve produzir requisitos a serem considerados na construção de softwares educacionais.

Capturar com precisão os requisitos do sistema é importante para o bom desenvolvimento de um produto de software e para o resultado geral de um projeto. Falhas na identificação, definição e validação dos requisitos podem fazer com que todo o ciclo de vida de um projeto seja comprometido. A Engenharia de Requisitos (ER) pode ser conceituada como o amplo espectro de tarefas e técnicas que levam a um entendimento dos requisitos na Engenharia de Software (ES) (Pressman e Maxim, 2016).

Por meio da ER é possível entender o que o usuário deseja, analisando suas necessidades, negociando uma solução para o problema, especificando e gerindo os requisitos durante o prosseguimento do projeto. Sem a ER há probabilidade da solução proposta não satisfazer as necessidades dos usuários. Sendo assim, a ER também tem interesse na qualidade que a solução computacional proporcionará ao seu usuário (Pressman e Maxim, 2016).

Outro processo que também busca aumentar a qualidade de soluções computacionais é o Design de Interação (DI). O DI está relacionado com a qualidade de uso de soluções computacionais. O objetivo do DI é dar formas as coisas digitais para o uso das pessoas (Lowgren, 2014). A ER e o DI possuem métodos, técnicas, modelos e ferramentas sistemáticas próprias para a construção de uma solução computacional. Uma das ferramentas para o DI é o framework SPIDe, proposto por Rosa e Matos (2016) e expandido por Pita et al. (2017).

Os estudos sobre o SPIDe (Rosa e Matos, 2016; Pita et al. 2017) tem tratado especificamente sobre requisitos ligados diretamente a Interação Humano-Computador (IHC), deixando de lado outros tipos de requisitos que podem estar relacionados a Engenharia de Software (ES). Desse modo, este artigo apresenta algumas evidências com o objetivo de verificar se a aplicação do SPIDe pode produzir requisitos de software e de interação de modo integrado. Para atingir esse objetivo está sendo realizada uma revisão sistemática da literatura e um estudo de caso.

As evidências resultantes a partir da análise inicial da revisão sistemática de literatura apontam para uma possível contribuição do SPIDe na concepção de requisitos de alinhados a ES e a IHC de softwares educacionais, de modo integrado.

\section{SPIDe}

O SPIDe ${ }^{1}$ é um framework semioparticipativo para o design de interação de artefatos computacionais (Rosa e Matos, 2016). A expressão "semioparticipativo" é formada pela união da partícula "semio", que faz referência a Semiótica; e da partícula "participativo", que faz referência a abordagens participativas (Baranauskas, 2013). A partir da perspectiva de design de interação semioparticipativo o SPIDe foi construído

\footnotetext{
1 (Rosa e Matos, 2016; Pita et al, 2017).
} 
VI Congresso Brasileiro de Informática na Educação (CBIE 2017)

Anais dos Workshops do VI Congresso Brasileiro de Informática na Educação (WCBIE 2017)

sob a base teórica da Engenharia Semiótica (EngSem) e dos princípios do Design Participativo (DP) (Rosa e Matos, 2016).

A EngSem é uma teoria de IHC de base semiótica que compreende o processo de interação humano-computador como um processo comunicativo entre o designer de interação e o usuário mediado pela interface computacional (De Souza, 2005). Por sua vez, o DP é uma abordagem que valoriza a participação dos (potenciais) usuários durante toda a concepção de uma tecnologia, onde para além de ser consultado, ele deve auxiliar na construção de soluções atuando em colaboração com o desenvolvedor (Luck, 2003).

O design de interação é um processo social que deve incluir não só o designer, mas também o usuário (Baranauskas, 2013), por isso, além da visão teórico-conceitual da EngSem, o SPIDe inclui técnicas participativas seguindo os princípios do DP (Rosa e Matos, 2016). A combinação da EngSem com o DP proposta pelo SPIDe transforma o paradigma inicial de interação humano-computador da EngSem, pois os usuários assumem o papel de designer (Rosa e Matos, 2016).

O SPIDe implementa o Design Centrado na Comunicação (DCC), uma prática da EngSem que considera o design de interação como um processo de manipulação de mensagens. O DCC divide o processo de design de interação em três etapas: análise de contexto, engenharia de interface e avaliação. Cada uma dessas etapas é composta por técnicas do DP e apesar de serem apresentadas sequencialmente, conforme a Figura 1, caso seja necessário, é possível retornar a técnicas ou etapas anteriores (Rosa e Matos, 2016).

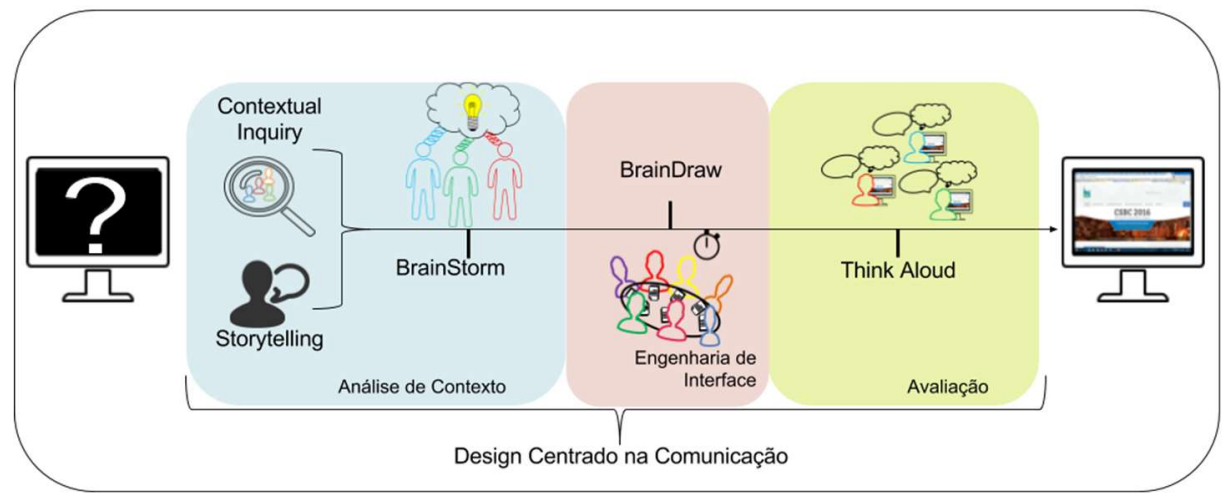

Figura 1. Composição do SPIDe (Pita et al., 2017).

A análise de contexto tem como objetivo conhecer o usuário, o seu contexto e como soluciona o problema. Essa etapa é composta por três técnicas participativas, o contextual inquiry, o storytelling e o brainstorm (Pita et al., 2017). De acordo com Rosa e Matos (2016), os resultados dessa etapa apresentam diretrizes sobre tecnologias disponíveis ao usuário, o modo como ele resolve o problema, como evitar problemas de interação e requisitos de interação.

A etapa de engenharia de interface tem por objetivo a criação de protótipos da tecnologia digital a ser (re)construída a partir da análise de contexto. O braindraw é a técnica que compõe essa etapa e resulta em desenhos com a fusão de ideias dos seus participantes que posteriormente deve basear a construção de protótipos. Por fim, a 
etapa de avaliação tem o propósito de avaliar os protótipos construídos pela etapa de engenharia de interface. Essa etapa é composta pela técnica think-aloud. Durante a aplicação dessa técnica os usuários devem verbalizar os seus pensamentos ao interagir com o protótipo criado (Rosa e Matos, 2016).

A primeira versão do SPIDe ( $c f$. Rosa e Matos, 2016) foi composta por quatro técnicas do DP (contextual inquiry, brainstorm, braindraw e think-aloud) e foi aplicada no domínio educacional, com estudantes de ensino fundamental e professores para o redesign de interação da rede socioeducacional TecCiencia ${ }^{2}$. A proposta do SPIDe é considerar o design por todos e para todos. Nessa versão inicial, Rosa e Matos (2016b) se dedicaram à aspectos culturais no design de interação. Para os pesquisadores, o SPIDe pode considerar esses aspectos devido a sua característica semioparticipativa.

A pesquisa desenvolvida por Pita et al. (2017) continuou o desenvolvimento do SPIDe. Aplicou-se a análise de contexto do SPIDe no domínio de mobilidade urbana com usuário com deficiência visual também a partir da perspectiva de design para todos e por todos. A pesquisa resultou na primeira modificação do SPIDe. Pita et al. (2017) sugerem a adição da técnica storytelling devido usuários cegos ou deficientes visuais (público alvo dos pesquisadores) sentirem-se constrangidos pela aplicação do contextual inquiry. Desse modo, é possível escolher entre a aplicação do contextual inquiry ou do storytelling durante o (re)design de interação de uma tecnologia digital interativa sob a perspectiva de uso/design para todos e por todos.

\subsection{Integração SPIDe \& ER}

Segundo Pressman e Maxim (2016), a ER é uma ação da Engenharia de Software, que estabelece uma base sólida para o projeto e construção de um software, fornecendo meios para entender as necessidades do usuário. A ER é composta por quatro fases: (i) elicitação, (ii) análise e negociação, (iii) documentação, e (iv) verificação e validação (Kontoya e Sommerville, 1998).

Durante a fase de elicitação de requisitos busca-se obter o máximo de informações importantes e relevantes de um determinado produto para descobrir seus requisitos (Kontoya e Sommerville, 1998). Tendo em vista que o objetivo da fase de elicitação assemelha-se com o propósito das etapas de análise de contexto e engenharia de interface do SPIDe, propõe-se que essas etapas do SPIDe possam contribuir com essa fase da ER.

A fase de verificação e validação dos requisitos deve ser realizada com o objetivo de verificar possíveis omissões, conflitos e ambiguidades nos requisitos descritos, validando-os (Kontoya e Sommerville, 1998). O objetivo dessa fase é análogo à etapa de avaliação do SPIDe, visto que ambos buscam identificar se o que foi criado por meio das fases/etapas anteriores está conforme as necessidades e desejos dos usuários.

\footnotetext{
${ }^{2}$ www.tecciencia.ufba.br
} 
VI Congresso Brasileiro de Informática na Educação (CBIE 2017)

Anais dos Workshops do VI Congresso Brasileiro de Informática na Educação (WCBIE 2017)

A partir dessas relações foi possível criar a seguinte hipótese: o SPIDe pode propiciar a ER de forma integrada quando suas etapas são aplicadas em analogia com as fases da ER.

\section{Metodologia}

De acordo com Rosa e Matos (2016) e Pita et al. (2017), a aplicação do SPIDe pode gerar requisitos para o (re)design de interação considerando aspectos culturais, a participação de crianças, adultos e deficientes visuais nos domínios educacional e de mobilidade urbana. A pesquisa em andamento apresentada neste artigo tem como objetivo expandir o SPIDe verificando se a sua aplicação pode gerar requisitos de software e de interação de modo integrado.

Para atingir esse objetivo está sendo realizada uma pesquisa de natureza qualiquanti com as seguintes ferramentas epistemológicas: revisão sistemática e estudo de caso; temas das próximas seções, sequencialmente. Até o momento da submissão foi realizada somente a revisão sistemática que resultou em um corpus de 10 produções científicas. O estudo de caso ainda será aplicado.

\section{Revisão Sistemática da Literatura}

A revisão sistemática deste trabalho tem como objetivo explorar produções científicas que apresentam resultados da utilização das técnicas contidas no SPIDe contribuindo com a geração de requisitos ou auxiliando as etapas do processo de engenharia de requisitos. Para guiar a revisão sistemática foi utilizado o procedimento proposto por Kitchenham (2004).

A revisão sistemática tem como objetivo, baseada em produções científicas anteriores, responder as seguintes questões de pesquisa:

(1) As técnicas do SPIDe têm sido utilizadas em conjunto com as atividades do processo de Engenharia de Requisitos?

(2) Em qual etapa do processo da engenharia de requisitos as técnicas do SPIDe têm sido utilizadas?

(3) As técnicas do SPIDe têm sido utilizadas para realizar a elicitação de requisitos de software?

(4) Quais tipos de requisitos são gerados por meio da utilização das técnicas do SPIDe quando aplicadas à Engenharia de Requisitos?

Para responder essas perguntas foram buscadas produções científicas em sete bibliotecas científicas digitais. A string ${ }^{3}$ de busca utilizada foi definida com base nas técnicas contidas no framework SPIDE associadas à Engenharia de Requisitos.

As buscas nas bibliotecas digitais resultaram uma amostra inicial de 370 produções contendo 22 em duplicidade, restando 348 produções, no qual foi necessário realizar a leitura dos títulos, resumos e palavras-chave com o objetivo de filtrar os

3 ("engenharia de requisitos" OR "requirement engineering") AND ("contextual inquiry" OR "storytelling" OR "braindraw" OR "braindrawing" OR "brainstorm" OR "brainstorming" OR "thinkaloud" OR "think aloud") 
VI Congresso Brasileiro de Informática na Educação (CBIE 2017)

Anais dos Workshops do VI Congresso Brasileiro de Informática na Educação (WCBIE 2017)

trabalhos que tivessem relação com o tema em questão, considerando os critérios de inclusão e exclusão definidos no planejamento.

Após a realização dessa etapa de seleção, 326 trabalhos foram rejeitados e restaram 22 como aceitos. Os trabalhos coletados na etapa de seleção foram lidos de forma integral com o objetivo de coletar somente trabalhos relevantes para realizar a condução da pesquisa. Através da aplicação desta etapa de extração foram aceitos 10 trabalhos e 12 trabalhos foram excluídos com base nos critérios de inclusão e exclusão definidos.

Foram excluídas produções com menos de três páginas (short papers), relatórios técnicos e livros (white papers), estudos secundários, produções que não apresentam resultados da utilização do contextual inquiry, storytelling, braindraw, brainstorm ou think-aloud relacionados ao processo de engenharia de requisitos de software e que não estejam escritos em português ou inglês. $\mathrm{O}$ diagrama da Figura 2 a demonstra de forma simplificada o processo de Revisão Sistemática, os resultados iniciais e finais.

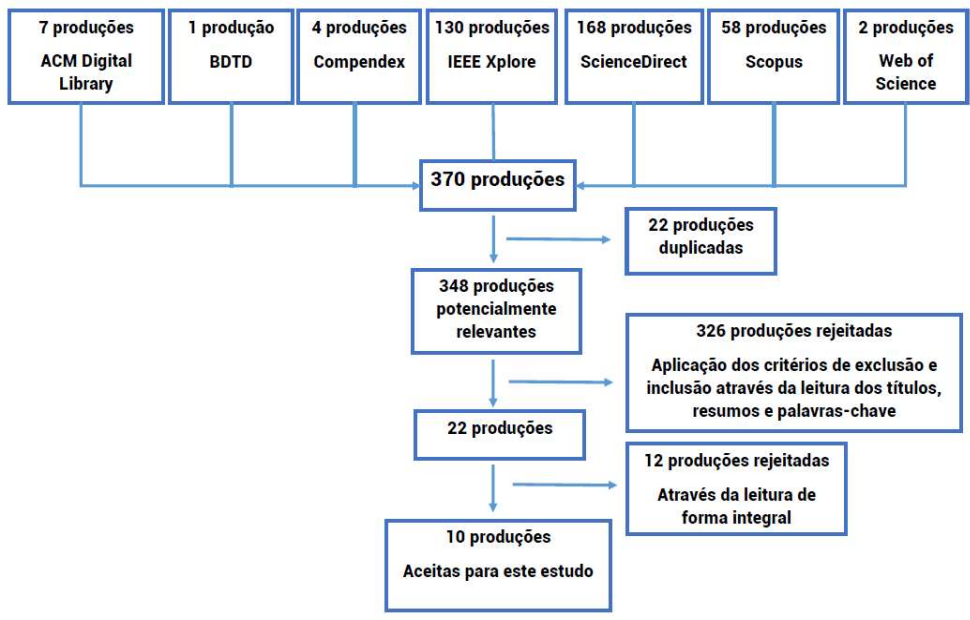

Figura 2. Detalhamento da Revisão Sistemática da Literatura.

Os 10 trabalhos ${ }^{4}$ resultantes da busca nas bibliotecas digitais e da aplicação dos critérios de inclusão e exclusão formam o corpus de produções científicas para responder as questões da revisão sistemática ( $c f$. Quadro 1).

Quadro 1. Produções científicas encontradas.

\begin{tabular}{|c|c|c|}
\hline Índice & Título & Ano \\
\hline P1 & $\begin{array}{c}\text { Selecting Creativity Techniques for Creative Requirements: An Evaluation of Four } \\
\text { Techniques using Creativity Workshops }\end{array}$ & 2015 \\
\hline P2 & A creative process to elicit contexts for context sensitive systems & 2015 \\
\hline P3 & $\begin{array}{c}\text { Assessment and Evaluation of Requirements Elicitation Techniques Using Analysis } \\
\text { Determination Requirements Framework }\end{array}$ & 2014 \\
\hline
\end{tabular}

${ }^{4}$ cf. (Lai, Peng e Ni, 2014; Batista e Silva, 2015; Besrour, Rahim e Dominic, 2014; Laporti, Boeges e Braganholo, 2009; Pasquale et al., 2013; Svensson e Taghavianfar, 2015; Sinnig et al., 2010; Boulila, Hoffmann e Herrmann, 2011; Sakhnini, Berry e Mich, 2010; Batista, 2014). 
VI Congresso Brasileiro de Informática na Educação (CBIE 2017)

Anais dos Workshops do VI Congresso Brasileiro de Informática na Educação (WCBIE 2017)

\begin{tabular}{|c|c|c|}
\hline P4 & $\begin{array}{c}\text { Collaborative Method for Business Process Oriented Requirements Acquisition and } \\
\text { Refining }\end{array}$ & 2014 \\
\hline P5 & Um processo criativo de descoberta de contextos para sistemas sensíveis a contexto & 2014 \\
\hline P6 & $\begin{array}{c}\text { Requirements Engineering Meets Physiotherapy: An Experience with Motion- } \\
\text { Based Games }\end{array}$ & 2013 \\
\hline P7 & $\begin{array}{l}\text { Using Storytelling to Record Requirements: Elements for an Effective } \\
\text { Requirements Elicitation Approach }\end{array}$ & 2011 \\
\hline P8 & $\begin{array}{c}\text { Validation of the Effectiveness of an Optimized EPMcreate as an Aid for Creative } \\
\text { Requirements Elicitation }\end{array}$ & 2010 \\
\hline P9 & $\begin{array}{c}\text { Structured Digital Storytelling for Eliciting Software Requirements in the ICT4D } \\
\text { Domain }\end{array}$ & 2010 \\
\hline $\mathrm{P} 10$ & Athena: A collaborative approach to requirements elicitation & 2009 \\
\hline
\end{tabular}

As produções científicas analisadas foram publicadas entre os anos de 2009 e 2015. Em sua maioria, elas apresentam estudos sobre a utilização de técnicas específicas junto ao processo de ER de software. Três delas são brasileiras, duas vinculadas a Universidade Federal de Pernambuco (Batista e Silva, 2015; Batista, 2014) e uma vinculada a Universidade Federal do Rio de Janeiro (Laporti, Borges e Braganholo, 2009).

Respondendo a primeira questão norteadora da revisão sistemática, somente duas técnicas do SPIDe têm sido utilizadas por pesquisadores em atividades do processo de ER - o Quadro 2 apresenta as técnicas do SPIDe utilizadas nas produções encontradas. As duas técnicas foram utilizadas pelos pesquisadores durante a fase de elicitação de requisitos, o que responde a segunda e a terceira questão norteadora da revisão sistemática. Todavia, não foi especificado quais tipos de requisitos foram coletados por meio de suas aplicações, deixando a quarta questão norteadora como lacuna.

Quadro 2. Técnicas do SPIDe utilizadas em cada produção científica.

\begin{tabular}{|c|c|c|c|c|c|c|c|c|c|c|c|}
\hline Técnica & P1 & P2 & P3 & P4 & P5 & P6 & P7 & P8 & P9 & P10 & SPIDe \\
\hline Storytelling & & $\mathrm{X}$ & & $\mathrm{X}$ & $\mathrm{X}$ & & $\mathrm{X}$ & & $\mathrm{X}$ & $\mathrm{X}$ & $\mathrm{X}$ \\
\hline $\begin{array}{c}\text { Contextual } \\
\text { inquiry }\end{array}$ & & & & & & & & & & & $\mathrm{X}$ \\
\hline Brainstorm & $\mathrm{X}$ & & $\mathrm{X}$ & & & $\mathrm{X}$ & & $\mathrm{X}$ & & & $\mathrm{X}$ \\
\hline Braindraw & & & & & & & & & & & $\mathrm{X}$ \\
\hline Think-aloud & & & & & & & & & & & $\mathrm{X}$ \\
\hline
\end{tabular}

Nas produções selecionadas não foram encontradas informações sobre o uso das técnicas contextual inquiry, braindraw e think-aloud, sendo assim, não foi possível identificar em quais etapas estas técnicas podem ser utilizadas junto ao processo de ER. 
VI Congresso Brasileiro de Informática na Educação (CBIE 2017)

Anais dos Workshops do VI Congresso Brasileiro de Informática na Educação (WCBIE 2017)

\section{Estudo de Caso}

O estudo de caso tem como objetivo investigar se o SPIDe pode produzir requisitos de software. Para isso, será estudada a aplicação do SPIDe durante a condução do processo de ER no desenvolvimento de um Ambiente Virtual de Aprendizagem (AVA) para a Biblioteca Pública Epifânio Dória em Aracaju/SE. A construção do AVA faz parte de um projeto de extensão vinculado à Universidade Federal de Sergipe intitulada "Uma proposta de Ambiente Virtual de Aprendizagem para disseminação da inclusão digital na sociedade sergipana".

As técnicas do SPIDe serão aplicadas nas fases de elicitação e, verificação e avaliação. Durante a fase de elicitação de requisitos serão aplicadas as técnicas contextual inquiry ou storytelling, brainstorm e braindraw. A aplicação dessas técnicas tem por objetivo a identificação de informações sobre as necessidades do usuário, a proposta de solução que será desenvolvida e como eles pensam que essa solução pode ser construída, o que reflete tanto nos objetivos da fase da ER, quanto nas etapas do SPIDe.

$\mathrm{Na}$ fase de verificação e validação dos requisitos será aplicada a técnica thinkaloud. Desse modo o usuário deverá verbalizar todos os seus pensamentos enquanto utilizam um protótipo construído a partir da concepção da solução computacional, permitindo a identificação de dúvidas, dificuldades, críticas e sugestões sobre o protótipo construído (Rosa e Matos, 2016).

Ao fim da execução do procedimento descrito, será realizada uma entrevista com os participantes da pesquisa a fim de coletar sugestões e identificar aspectos positivos e negativos da aplicação do SPIDe junto ao processo de ER. As informações resultantes desta entrevista, bem como o material resultante da aplicação das técnicas do SPIDe e da revisão sistemática serão sumarizados e analisados com o objetivo de responder à questão norteadora da pesquisa.

\section{Considerações Finais}

Este artigo apresentou resultados iniciais de uma pesquisa em andamento com o objetivo de expandir o framework SPIDe à Engenharia de Requisitos e a construção de um AVA. O SPIDe inicialmente foi desenvolvido para o (re)design de interação, todavia, com as pesquisas realizadas para a construção e desenvolvimento do SPIDe foi possível identificar que alguns dos seus resultados são requisitos de software. Desse modo, foi proposta a pesquisa para investigar a aplicabilidade do SPIDe à Engenharia de Requisitos.

Com os resultados iniciais da pesquisa foi possível identificar que algumas das técnicas que compõem o SPIDe, o storytelling e o brainstorm, foram utilizadas em estudos anteriores e podem gerar requisitos de software. Todavia, não foi possível identificar quais os tipos de requisitos são gerados e, para além disso, não foram encontradas a aplicação do contextual inquiry, braindraw e think-aloud deixando lacunas para a continuação da pesquisa.

As próximas etapas desta pesquisa são: a análise aprofundada dos artigos encontrados na Revisão Sistemática da Literatura e a execução do estudo de caso para o 
VI Congresso Brasileiro de Informática na Educação (CBIE 2017)

Anais dos Workshops do VI Congresso Brasileiro de Informática na Educação (WCBIE 2017)

desenvolvimento do AVA para a Biblioteca Epifánio Dória. Desse modo, com a conclusão dessa pesquisa será possível identificar se o SPIDe pode propiciar o processo de ER de software e também o levantamento de requisitos para o AVA.

\section{Agradecimentos}

Agradecemos aos participantes da pesquisa; ao Grupo de Pesquisa e Extensão em Informática, Educação e Sociedade - Onda Digital; e à CAPES pelo financiamento parcial da pesquisa.

\section{Referências}

Baranauskas, M. C. C. (2013). O modelo semioparticipativo de design. In: Codesign de Redes Digitais: tecnologia a serviço da inclusão social, M. C. C. Baranauskas, M. C. Martins and J. A. Valente (eds.). Penso.

Barbosa, S. and Silva, B. (2010). Interação Humano Computador. Elsevier, Rio de Janeiro.

Batista, C. (2014). Um Processo Criativo de Descoberta de Contextos para Sistemas Sensiveis a Contexto. Dissertação (Mestrado em Ciência da Computação). Universidade Federal de Pernambuco.

Batista, C. and Silva, C. (2015). A creative process to elicit contexts for context sensitive systems. In: Proceedings of the annual conference on Brazilian Symposium on Information Systems: Information Systems: A Computer SocioTechnical Perspective. Brazilian Computer Society.

Besrour, S., Rahim, L. and Dominic, P. (2014). Assessment and evaluation of requirements elicitation techniques using analysis determination requirements framework. In Computer and Information Sciences (ICCOINS), 2014 International Conference on. IEEE, 1-6.

Boulila, N., Hoffmann, A. and Herrmann, A. (2011). Using Storytelling to record requirements: Elements for an effective requirements elicitation approach. In: Multimedia and Enjoyable Requirements Engineering-Beyond Mere Descriptions and with More Fun and Games (MERE), 2011 Fourth International Workshop on. IEEE, 9-16.

De Souza, C. S. (2005). The Semiotic Engineering of Human-Computer Interaction. MIT Press.

Gomes, A. S. and Padovani, S. (2005). Usabilidade no ciclo de desenvolvimento de software educativo. In: Anais do Simpósio Brasileiro de Informática na Educação. SBC.

Gomes, A. S. and Wanderley, E. G. (2003). Elicitando requisitos em projetos de Software Educativo. In: Anais do IX Workshop de Informática na Escola. SBC, 119130.

Junior, O. O. B. and Paola, Yuska. (2014). Análise de Abordagens Objetivas para Avaliação de Softwares Educativos. In: Proceedings of the 13th Brazilian Symposium on Human Factors in Computing Systems. ACM, 353-356. 
VI Congresso Brasileiro de Informática na Educação (CBIE 2017)

Anais dos Workshops do VI Congresso Brasileiro de Informática na Educação (WCBIE 2017)

Kitchenham, B. (2004). Procedures for performing systematic reviews. Keele, UK, Keele University, 33(2004), 1-26.

Kotonya, G., and Sommerville, I. (1998). Requirements engineering: processes and techniques. Wiley Publishing.

Kujala, S. and Kauppinen, M. and Rekola, S. (2001) Bridging the Gap between User Needs and User Requirements. In: Proceedings of The Panhellenic Conference with International Participation in Human-Computer Interaction, IEEE, 45-50.

Lai, H., Peng, R. and Ni, Y. (2014). A collaborative method for business process oriented requirements acquisition and refining. In: Proceedings of the 2014 International Conference on Software and System Process. ACM, 84-93.

Laporti, L., Borges, M. and Braganholo, V. (2009). Athena: A collaborative approach to requirements elicitation. Computers in Industry 60, 6 (2009), 367-380.

Lowgren, J. (2014). Interaction Design - brief intro. In: The Encyclopedia of HumanComputer Interaction (2nd ed.), Mads Soegaard and Rikke Friis Dam (eds.). The Interaction Design Foundation, Aarhus, Dinamarca.

Luck, R. (2003). Dialogue in participatory design. Design Studies 24, 6: 523-535.

Pasquale, L., Spoletini, P., Pometto, D., Blasi, F. and Redaelli, T. (2013). Requirements engineering meets physiotherapy: An experience with motion-based games. In: International Working Conference on Requirements Engineering: Foundation for Software Quality. Springer, 315-330.

Pressman, R. and Maxim, B. (2016). Engenharia de Software. McGraw Hill Brasil, $8^{\text {a }}$ ed.

Pita, G. (2016). Design por todos: participação de deficientes visuais no codesign de interação humano-computador. Trabalho de Conclusão de Curso - Engenharia da Computação. Universidade Federal da Bahia.

Rosa, J. e Matos, E. (2016). Semio-participatory framework for interaction (re)design of education softwares. In: Proceedings of the 15th Brazilian Symposium on Human Factors in Computer Systems. (IHC'16).

Sakhnini, V., Berry, D. and Mich, L. (2010). Validation of the effectiveness of an optimized EPMcreate as an aid for creative requirements elicitation. In: International Working Conference on Requirements Engineering: Foundation for Software Quality. Springer, 91-105.

Sinnig, D., Pitula, K., Becker, R., Radhakrishnan, T. and Forbrig, P. (2010). Structured digital storytelling for eliciting software requirements in the ICT4D domain. In: Human-Computer Interaction. Springer, 58-69.

Sommerville, I. (2007). Engenharia de Software. Pearson Addison-Wesley, 8 a ed.

Svensson, R. and Taghavianfar, M. (2015). Selecting creativity techniques for creative requirements: An evaluation of four techniques using creativity workshops. In: Requirements Engineering Conference (RE), 2015 IEEE 23rd International. IEEE, $66-75$. 